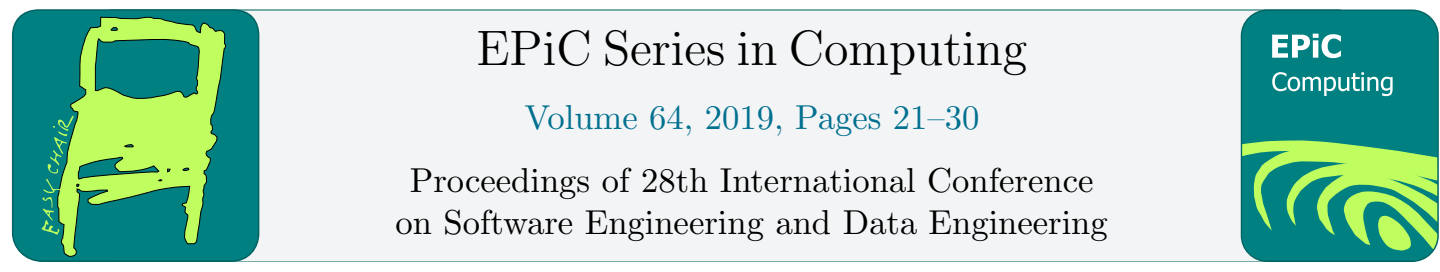

\title{
Design and Development of the CTAR All-Star
}

\author{
Terri Heglar, Andrew Penrose, Austin Yount, \\ Kristine Galek, Yantao Shen, Sergiu M. Dascalu, and Frederick C. Harris, Jr. \\ University of Nevada Reno, NV 89557, USA \\ theglar@nevada.unr.edu apenrose@nevada.unr.edu austinyount11@gmail.com \\ kgalek@med.unr.edu ytshen@unr.edu dascalus@cse.unr.edu fred.harris@cse.unr.edu
}

\begin{abstract}
The CTAR All-Star is a system consisting of a rubber ball, a pressure sensor, and a bluetooth transmitter paired with a cross-platform mobile application. The device is used as a rehabilitation tool for people with dysphagia in a similar fashion to the traditional chin tuck against resistance (CTAR) exercise by squeezing a ball between the chin and upper chest. The mobile device monitors and displays the pressure inside the ball on a real-time graph allowing the patient to follow exercise routines set by Speech-Language Pathologists. Additionally, the application stores exercise data that can be used to both monitor the patient's progress over time and provide objective data for future research purposes.
\end{abstract}

keywords: chin tuck against resistance, dysphagia, rehabilitation.

\section{Introduction}

Dysphagia is defined as swallowing difficulty that can result in serious health consequences including malnutrition, dehydration, aspiration pneumonia, and death [5]. Dysphagia may be caused by behavioral, sensory, or motor deficits or a combination of deficits. Dysphagia is a symptom of a primary medical condition caused by neurological, subcortical, and/or neuromuscular impairments. Approximately 37 percent of patients with these medical conditions are diagnosed with some degree of dysphagia in the US [1]. Therefore, it can be estimated that approximately 3.3 million people undergo medical treatment for dysphagia for various conditions each year. For the purposes of this paper, the focus will be on dysphagia caused by upper esophageal sphincter (UES) dysfunction. One common exercise for increasing the strength of the suprahyoid muscles which increases the size of the upper esophageal sphincter opening during the swallow is the chin tuck against resistance (CTAR). CTAR is a known treatment for dysphagia caused by UES dysfunction [9]. The CTAR All-Star aims to improve on the traditional rehabilitation experience for patients and Speech-Language Pathologists (SLPs). The system capitalizes on the increasing prevalence of mobile smartphones and tablets in modern society to create a package that is both affordable and feature-rich. The CTAR All-Star is composed of a cross-platform mobile application and a wireless exercise device that can be used by both parties for creating, assigning, and performing exercise routines as well as monitoring the results of rehabilitation sessions. Using this system, The SLP can create personalized exercise 
routines and assign them to patients. When performing an exercise routine, the application guides the patient through the exercise step by step while plotting the pressure inside the ball in real time.

The rest of this paper is structured as follows: Section 2 discusses the software design of the mobile application, Section 3 goes over the hardware design of the exercise device, Section 4 walks through the implementation of the CTAR All-Star, and Section 5 reviews the conclusions and future work associated with the CTAR All-Star.

\section{Software Design}

The Software Design will discuss many of the requirements and use cases, the traceability matrix, and the database design of the application. The requirements and use cases for the application were realized through interviews with multiple stakeholders including SLPs.

\subsection{Functional Requirements}

Functional Requirements are "statements of services the system should provide, how the system should react to particular inputs, and how the system should behave in particular situations. In some cases, the functional requirements may also explicitly state what the system should not do" [6]. The following functional requirements describe the behavior of the CTAR All-Star.

1. The CTAR All-Star stores a user profile.

2. The CTAR All-Star identifies users only by EMR numbers.

3. The CTAR All-Star forces the user to log in for the first time.

4. The CTAR All-Star connects via Bluetooth 4.0 using an HM-10

5. The CTAR All-Star measures the gauge pressure between the ambient air and the inside of the ball.

6. The CTAR All-Star broadcasts pressure readings at least once every $100 \mathrm{~ms}$.

7. The CTAR All-Star allows the user to drop/change the current bluetooth connection.

8. The CTAR All-Star allows the user to start a new session.

9. The CTAR All-Star allows the user to stop the current session.

10. The CTAR All-Star offers Isometric and Isotonic exercise routines.

11. The CTAR All-Star provides a visual representation of the pressure during an exercise.

12. The CTAR All-Star stores the 1-Rep Max value used for calculating a threshold.

13. The CTAR All-Star stores a repetition count value.

14. The CTAR All-Star allows the user to view data from previous sessions.

15. The CTAR All-Star provides a countdown timer.

16. The CTAR All-Star timer pauses and restarts if the pressure falls below the threshold.

17. The CTAR All-Star allows the user to view aggregate data. 


\subsection{Non-functional Requirements}

Non-functional requirements are "constraints on the services or functions offered by the system. They include timing constraints, constraints on the development process, and constraints imposed by standards. Non-functional requirements often apply to the system as a whole rather than individual system features or services" [6]. The following list contains the Non-Functional requirements for the CTAR All-Star application.

1. The CTAR All-Star runs on Android and iOS platforms.

2. The CTAR All-Star communicates between the device and ball via Bluetooth 4.0.

3. The CTAR All-Star is implemented using the Xamarin framework.

4. The CTAR All-Star is written in C, C\#, XAML

5. The CTAR All-Star provides Visual feedback in real time.

6. The CTAR All-Star has a user friendly interface.

7. The CTAR All-Star protects the security of each user.

8. The CTAR All-Star visualization is neutral to appeals to multiple demographics.

\subsection{Use Cases}

Use cases describe on a high-level how the users should interact with an application. Figure 1 shows the interaction between the CTAR All-Star and the patient or doctor.

1. CreateUser: Patients and medical professionals should be able to make a patient user account from a mobile device using the patient's EMR number. Medical professionals should be able to make an account which allows them to view their patient's information.

2. LogIn: Patients should be able to log in to the mobile app the first time the app opens. The home screen is displayed after successful login.

3. PairDevice: Users should be able to connect their mobile device to the exercise ball by selecting from a list of available Bluetooth devices. The app will prompt this when a device is not connected or allow the user to change the connection through settings.

4. CreateExercise: Medical professionals should be able to make a patient exercise plan that the patient can work on between appointments. Plans would include specifying the exercise module and the number of times or frequency that the module should be completed during a specified time period.

5. Set1_RepMax: The app guides the patient through setting a 1-rep max at the beginning of each exercise session. The app prompts the user to squeeze the ball as hard as they can and stores the maximum pressure inside the ball.

6. PerformExercise: Patients should be able to follow an exercise routine. The program sets various pressure thresholds based on the patients 1 rep max. The program then guides the patient through the exercise routine where the patient squeezes and releases the ball while a counter counts the repetitions for a certain threshold.

7. SaveExercise: The patient's exercise should be saved at the end of a session so that the results can be viewed at a later date. 


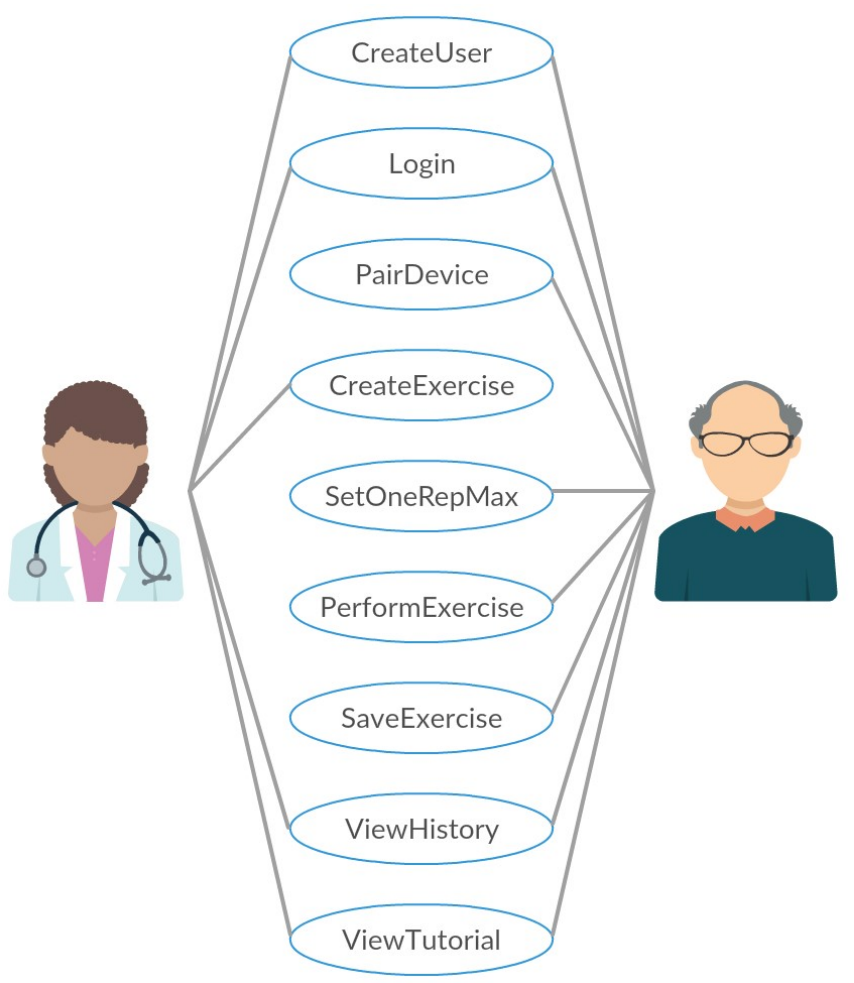

Figure 1: The Use Case Diagram shows the patient and doctor interactions with the CTAR All-Star.

8. ViewHistory: Patients and medical professionals should be able to view historical exercise data.

9. ViewTutorial: The patient or medical professional should be able to view various tutorials with screenshots instructing them on how to use the various features of the app.

\subsection{Traceability Matrix}

Traceability policies "define the relationships between each requirement and between the requirements and the system design that should be recorded.[6]" The CTAR All-Star Traceability Matrix, as seen in Figure 2, is the tool used to track these relationships. Organizing these connections is essential in analyzing proposed changes and the impact they have on other parts of the system.

\subsection{Database Design}

The main data structures used for the CTAR All-Star are tables in a SQLite[2] local database. Each patient is only identifiable by their Electronic Medical Record (EMR) number to avoid any HIPAA violations. The EMR is used along with their password to login when the patient initially opens the application. Table 1 depicts the class tables, the primary keys, and the 


\begin{tabular}{|c|c|c|c|c|c|c|c|c|c|}
\hline \multicolumn{10}{|c|}{ Use Cases } \\
\hline \multirow{18}{*}{ 号 } & & 1 & 2 & 3 & 4 & 5 & 6 & 7 & 8 \\
\hline & 1 & $\mathrm{x}$ & $\mathrm{X}$ & & & & & & \\
\hline & 2 & $\mathrm{x}$ & & & & & & & $\mathrm{x}$ \\
\hline & 3 & $\mathrm{X}$ & $\mathrm{x}$ & & & & & & \\
\hline & 4 & & & $\mathrm{x}$ & & & & & \\
\hline & 5 & & & & 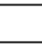 & $\mathrm{x}$ & $\mathrm{X}$ & $\mathrm{X}$ & \\
\hline & 6 & & & & & & $\mathrm{x}$ & $\mathrm{x}$ & \\
\hline & 7 & & & $\mathrm{X}$ & & & & & \\
\hline & 8 & & & & & $\mathrm{X}$ & $\mathrm{X}$ & $\mathrm{x}$ & \\
\hline & 9 & & & & & $\mathrm{X}$ & $\mathrm{X}$ & $\mathrm{X}$ & \\
\hline & 10 & & & & $\mathrm{X}$ & & $\mathrm{X}$ & $\mathrm{x}$ & \\
\hline & 11 & & & & & $\mathrm{x}$ & $\mathrm{x}$ & $\mathrm{x}$ & \\
\hline & 12 & & & & & $\mathrm{X}$ & & & \\
\hline & 13 & & & & $\mathrm{x}$ & & $\mathrm{x}$ & $\mathrm{x}$ & \\
\hline & 14 & & & & & & & & $\mathrm{x}$ \\
\hline & 15 & & & & & & $\mathrm{x}$ & $\mathrm{x}$ & \\
\hline & 16 & & & & & & $\mathrm{X}$ & $\mathrm{x}$ & \\
\hline & 17 & & & & & & & & $\mathrm{X}$ \\
\hline
\end{tabular}

Figure 2: The Traceability Matrix shows the relationships between each functional requirement and use case.

Table 1: Data Structures

\begin{tabular}{|l||l|l|}
\hline Class & Key & Attributes \\
\hline \hline User & Id & $\begin{array}{l}\text { Username, Password, userType, isLoggedIn, } \\
\text { OneRepMax, Session, DocID }\end{array}$ \\
\hline GraphMeasurement & Id & Time, Pressure \\
\hline Measurement & Id & $\begin{array}{l}\text { UserName, DocID, SessionNumber, TimeStamp, } \\
\text { DisplayTime, DisplayDate, Pressure, OneRepMax }\end{array}$ \\
\hline NativeDevice & Id & Name \\
\hline Patient & PatientId & PatientEmrNumber, DoctorName \\
\hline Tutorial & Id & Topic, Description, ImageName, isVisible, URL \\
\hline Workout & WorkoutId & $\begin{array}{l}\text { WorkoutName, PatientEmrNumber, DoctorID, } \\
\text { NumReps, NumSets, ThresholdPercentage, Hold- } \\
\text { Duration, RestDuration, Type }\end{array}$ \\
\hline
\end{tabular}

class attributes. The User class is role-based with their role being stored in the userType attribute. The UserName is the patient's EMR or another unique identifier for the doctors. An auto generated unique Id serves as the primary key. The DocID identifies the patient's corresponding SLP.

There is a one-to-many relationship between the User table and the Workout table. In the Workout table, there is a WorkoutID, WorkoutName, PatientEmrNumber, DoctorID, and several other attributes that allow the details of the workout to be stored, such as the number of reps, sets, and the duration of each step in the exercise. The WorkoutID specifies and uniquely identifies the workout number. The PatientEmrNumber is used to link each workout to the corresponding patient. The DoctorId corresponds to the proper medical professional. 
Additionally, there is a Measurement table. This table is used to record the measurement data from each exercise session. The measurement table contains the Id, UserName, DocID, SessionNumber, a timestamp with various display formats, Pressure, and OneRepMax. The Id is the primary key which is auto generated and uniquely identifies measurements from the ball. UserName and DocID are linked to the User table in a one-to-many relationship. The pressure field is used to record the pressure reading as an integer. The OneRepMax is used to track the patient's current capabilities at the time the exercise was completed.

The tables are related to display the history inside the application for both the patient and the medical professionals to review. If a medical professional's UserName is linked with a patient's DocID, then they have access to that patient's session history. The Patient class is designed to assist in this linkage, specifically for allowing a doctor to create workouts for a patient before they have created their own account. By linking the tables, the medical professional is able to analyze a patient's data and determine if they are making positive progress. If the patient is not making progress, a workout adjustment may be needed.

The remaining tables, GraphMeasurement, NativeDevice, and Tutorial are used for various other specific features in the application. The GraphMeasurement stores the data that will be displayed in the graph on the exercise screen. The NativeDevice stores the available bluetooth devices used when connecting to the hardware. Finally, Tutorial contains the individual instruction guides for the Tutorial's page.

\section{Hardware Design}

A major goal of the hardware was to make it affordable. A cost-effective device will enable patients to purchase their own personal device and perform exercises from within their home. Figure 3 shows the initial prototype and the hardware design can be seen in Figure 4 . The patient uses the device by squeezing the pneumatic ball between the chin and upper chest. A pressure transducer outputs an analog voltage to the Arduino before the analog reading is converted to digital and transmitted over Bluetooth 4.0. The hardware components used in the prototype include the following:

- Pneumatic ball: This ball is appropriately sized to fit between an adults chin and upper chest. It has a valve for an inflation needle which makes probing the internal pressure simple.

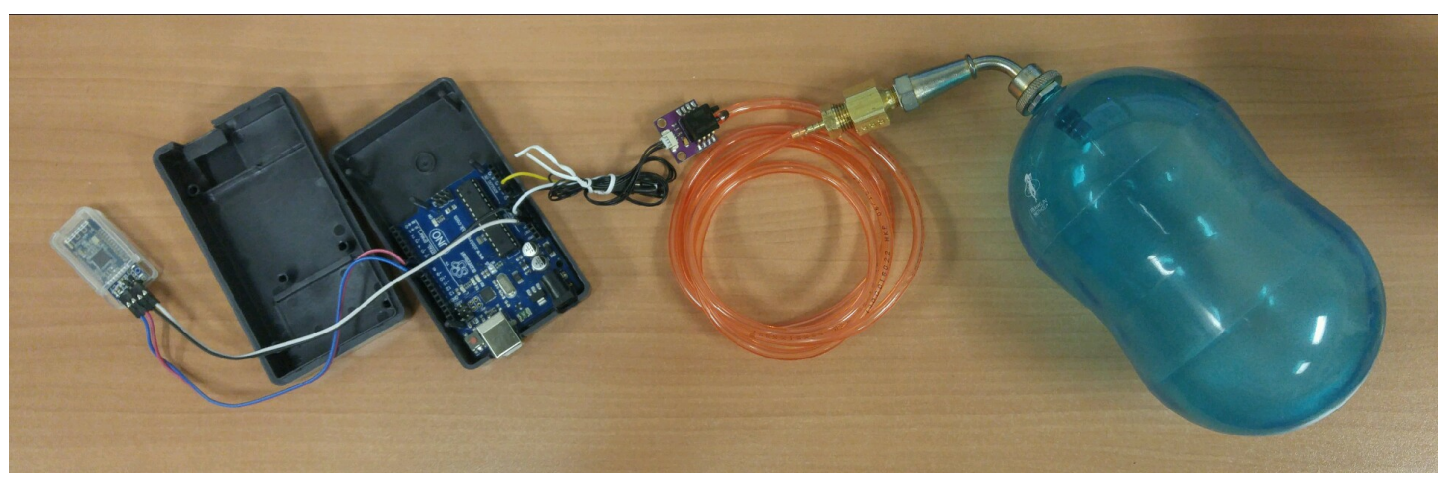

Figure 3: The initial prototype of the CTAR All-Star was powered by a PC through a USB cable. 


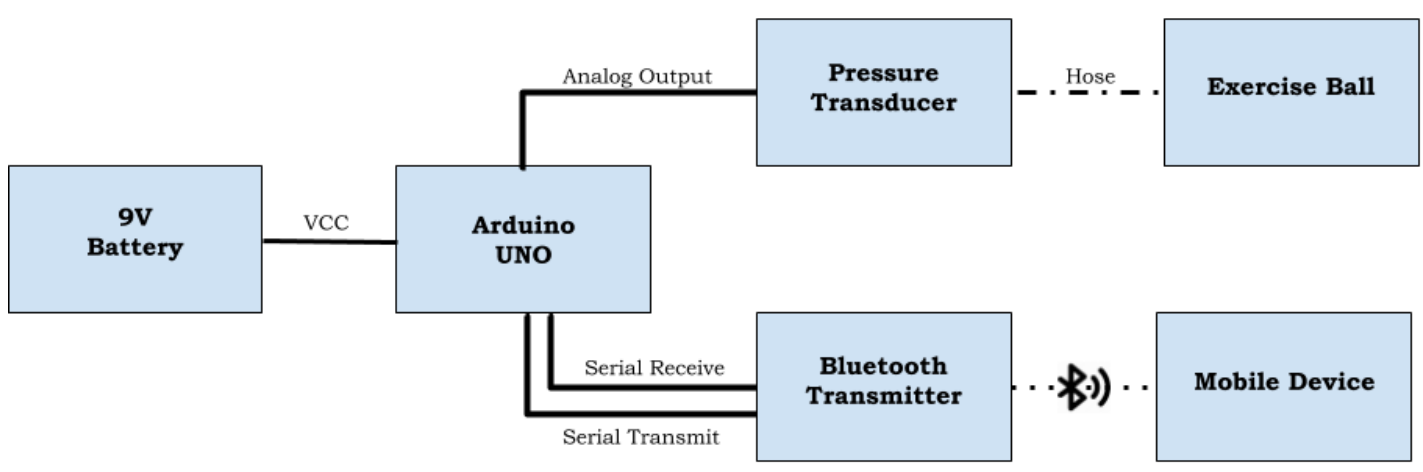

Figure 4: The Hardware Diagram defines how the components connect and interact.

- Inflation needle and plumbing: The inflation needle and plumbing allow the pressure sensor to read the pressure inside the ball.

- Pressure Transducer: The pressure transducer reports the gauge pressure inside of the ball as an analog voltage value between 0 and 4.5 volts. The pressure transducer used in the prototype has a range of 0 to $7.25 \mathrm{psi}$.

- Arduino Uno: The Arduino Uno[7] reads the analog pressure from the pressure transducer every $50 \mathrm{~ms}$. The analog value is converted to digital and then sent to the Bluetooth transmitter via universal asynchronous receiver/transmitter (UART).

- Bluetooth Transmitter: The Bluetooth transmitter receives readings via UART and then transmits them to the mobile device.

The pressure inside the ball is sampled continuously and each reading is transmitted over Bluetooth 4.0 to the mobile app where the pressure is recorded and displayed for the user. Figure 5 shows the device in action.

\section{Software Implementation}

The CTAR All-Star is designed with a user friendly interface. It allows doctors to create or modify exercise plans (Figure 6) while patients can complete their prescribed exercises with visual feedback (Figure 7). The exercise history is stored for monitoring improvements and will additionally be used for future research purposes (Figure 8). It is a cross platform application, working on both Android and iOS tablets and mobile devices (Figure 9).

The mobile application is built using the Xamarin.Forms[3] framework in the Microsoft Visual Studio IDE. All of the code is written using a combination of C\# and XAML, the Plugin.BLE package is utilized for management of bluetooth 4.0 connections, the Syncfusion[8] plugin is utilized for plotting the ball pressure, and the mobile app is built for use on both iOS and Android devices. 


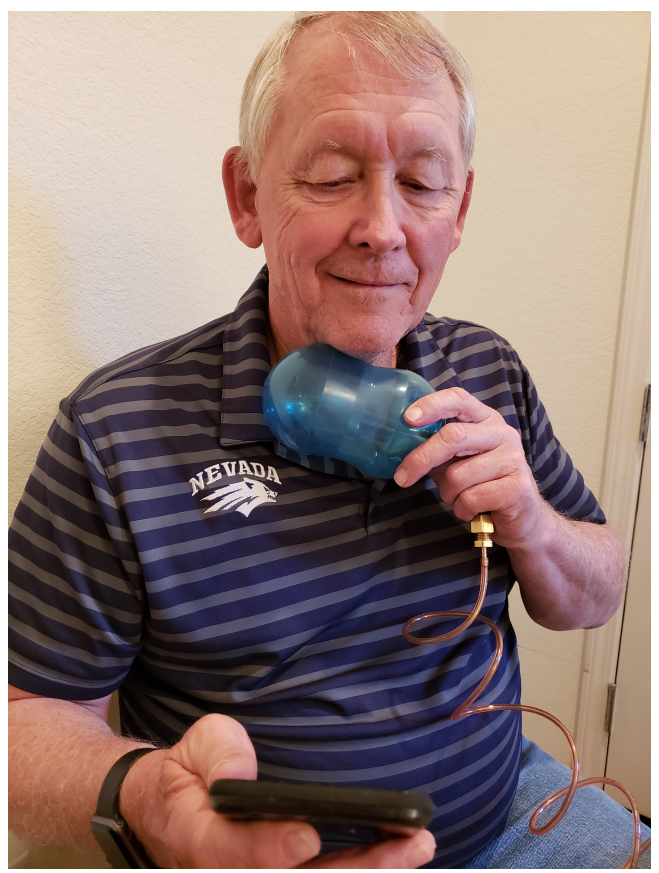

Figure 5: A patient learns how to use the CTAR All-Star.

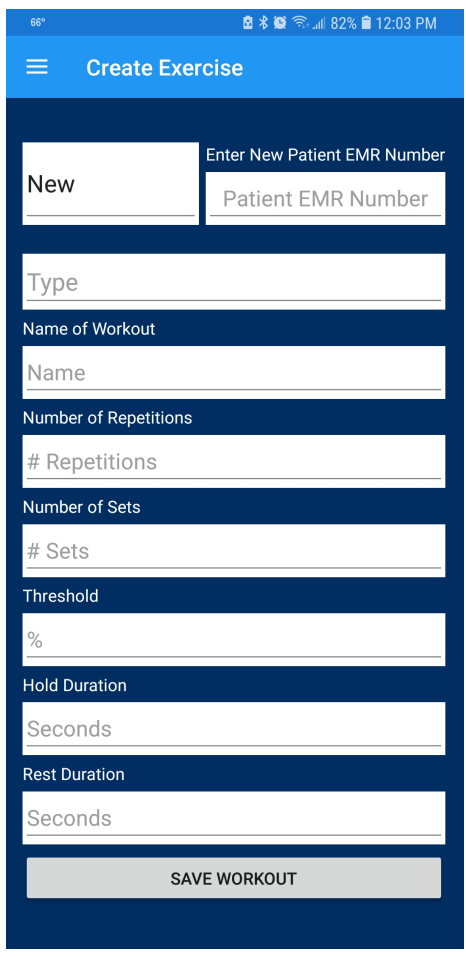

Figure 6: Doctors can create an exercise.
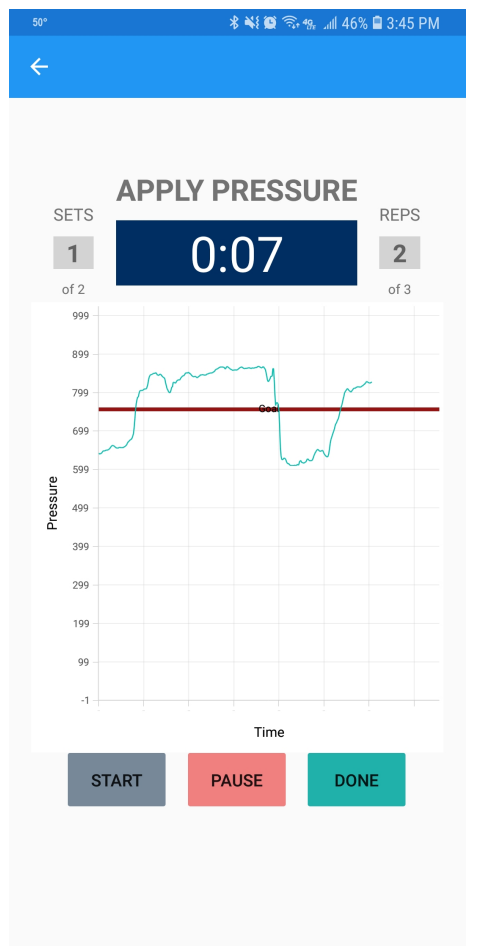

Figure 7: Patients are led Figure 8: History is stored for through the exercise.

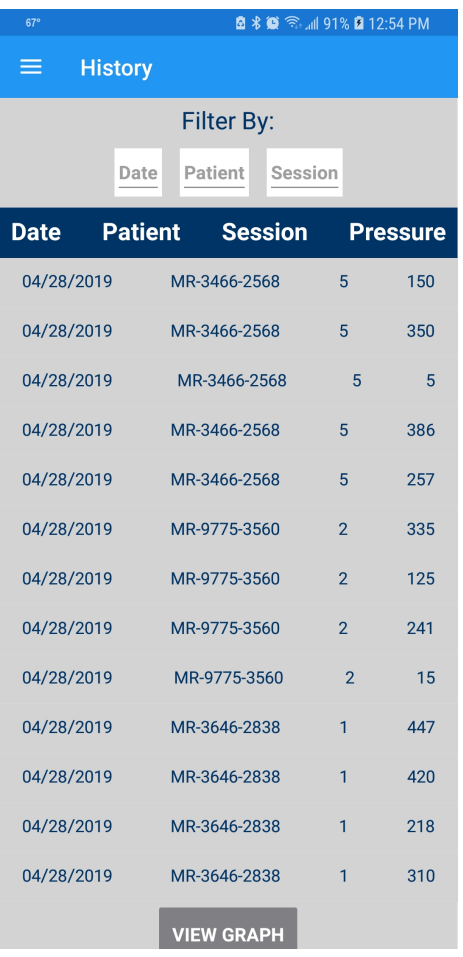

future research. 


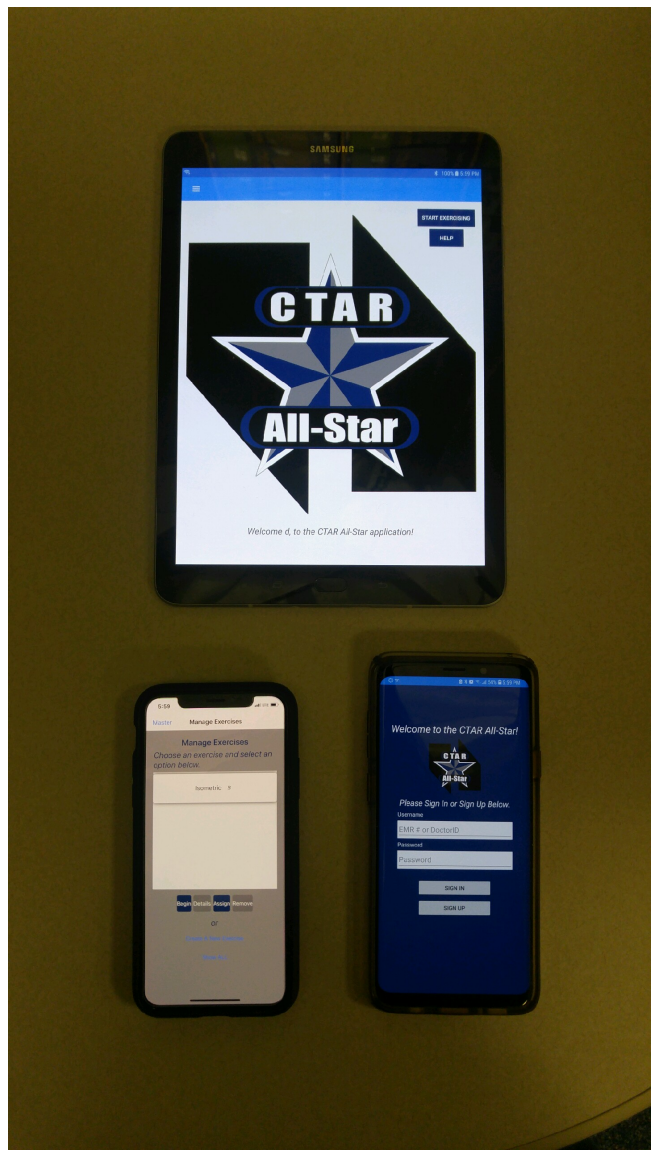

Figure 9: The CTAR All-Star can be run on multiple devices and operating systems including Android and Apple's iOS.

\section{Conclusions and Future Work}

\subsection{Conclusions}

The CTAR All-Star allows medical professionals and patients with dysphagia to track CTAR workouts over time. This application is significant because with technology incorporated into the ball, doctors are able to accurately track patient progress. The real time graph is interactive, gives instant feedback, and guides patients through their workouts. This first-of-it's-kind crossplatform application is necessary to gauge the success of both the CTAR exercise and the rehabilitation progress of the patient. With future work such as an online server and embedding the hardware inside of the ball, the CTAR All-Star can become a marketable product.

\section{$5.2 \quad$ Future Work}

This software can be modified to be used with different types of devices such as expiratory/inspiratory muscle trainers, IOPI ${ }^{\circledR}$, or SEMG. Although aimed at medical rehabilitation exercises, it may also be useful in other fields. This specific project could also integrate a game 
to make rehabilitation exercises more fun and interactive. Creating a mobile game, or incorporating a game already created for dysphagia rehabilitation, such as Avaler's Adventure, to work inside the CTAR mobile application would be a great option [4]. Additionally, a server hosted database back end with an API would make communication across multiple devices possible. Finally, refining the design of the hardware so that the electronics can be embedded inside the ball would greatly improve the reliability and ease of use of the device.

\section{Acknowledgments}

This material is based upon work supported by the National Science Foundation under grant number IIA1301726. Any opinions, findings, and conclusions or recommendations expressed in this material are those of the authors and do not necessarily reflect the views of the National Science Foundation.

\section{References}

[1] Neil Bhattacharyya. The prevalence of dysphagia among adults in the united states. Otolaryngology-Head and Neck Surgery, 151(5):765-769, 2014. PMID: 25193514.

[2] SQLite Consortium. About SQLite. https://www.sqlite.org/about.html. Accessed: 2019-06-26.

[3] Charles Petzold. Creating Mobile Apps with Xamarin.Forms. Microsoft Press, 2016.

[4] Catherine R. Pollock, Daneil A. Lopez, Wambaugh Gunnar, Luis Almanzar, Amanda Morrissey, Kathryn Krings, Kristine Galek, and Frederick C. Harris Jr. Avaler's adventure: an open source game for dysphagia therapy. Proceedings of the ISCA 26th International Conference on Software Engineering and Data Engineering (SEDE 2017), 26:25-30, 2017.

[5] Nicole Rogus-Pulia, Georgia Malandraki, Joanne Robbins, and Sterling Johnson. Understanding dysphagia in dementia: The present and the future. Current physical medicine and rehabilitation reports, 3:86-97, 012015.

[6] Ian Sommerville. Software Engineering. Pearson, 2016.

[7] Arduino S.r.l. Arduino language reference. https://www.arduino.cc/reference/en/. Accessed: 2019-06-26.

[8] Syncfusion. Syncfusion.com. https://www.syncfusion.com/. Accessed: 2019-06-26.

[9] Wai Lam Yoon, Jason Kai Peng Khoo, and Susan J. Rickard Liow. Chin tuck against resistance (ctar): New method for enhancing suprahyoid muscle activity using a shaker-type exercise. Dysphagia, 29(2):243-248, Apr 2014. 